\title{
Myeloid-specific targeting of Notch ameliorates murine renal fibrosis via reduced infiltration and activation of bone marrow- derived macrophage
}

\author{
Yali Jiang ${ }^{1,2}$, Yuanyuan Wang ${ }^{1,2}$, Pengfei Ma ${ }^{1}$, Dongjie An ${ }^{1}$, Junlong Zhao ${ }^{1}$, Shiqian Liang1, Yuchen Ye', \\ Yingying Lu ${ }^{2}$, Peng Zhang ${ }^{2}$, Xiaowei Liu ${ }^{2 \bowtie}$, Hua Han ${ }^{1 凶}$, Hongyan Qin ${ }^{1 凶}$ \\ ${ }^{1}$ State Key Laboratory of Cancer Biology, Department of Medical Genetics and Developmental Biology, Fourth Military Medical \\ University, Xi'an 710032, China \\ 2 Department of Nephrology, Xijing Hospital, Fourth Military Medical University, Chang-Le Xi Street \#15, Xi'an 710032, China \\ $\triangle$ Correspondence: liuxiaow@fmmu.edu.cn (X. Liu), huahan@fmmu.edu.cn (H. Han), hyqin@fmmu.edu.cn (H. Qin) \\ Received November 28, 2017 Accepted February 28, 2018
}

\begin{abstract}
Macrophages play critical roles in renal fibrosis. However, macrophages exhibit ontogenic and functional heterogeneities, and which population of macrophages contributes to renal fibrosis and the underlying mechanisms remain unclear. In this study, we genetically targeted Notch signaling by disrupting the transcription factor recombination signal binding protein-JK (RBP-J), to reveal its role in regulation of macrophages during the unilateral ureteral obstruction (UUO)-induced murine renal fibrosis. Myeloid-specific disruption of RBP-J attenuated renal fibrosis with reduced extracellular matrix deposition and myofibroblast activation, as well as attenuated epithelial-mesenchymal transition, likely owing to the reduced expression of TGF- $\beta$. Meanwhile, $R B P-J$ deletion significantly hampered macrophage infiltration and activation in fibrotic kidney, although their proliferation appeared unaltered. By using macrophage clearance experiment, we found that kidney resident macrophages made negligible contribution, but bone marrow (BM)-derived macrophages played a major role in renal fibrogenesis. Further mechanistic analyses showed that Notch blockade reduced monocyte emigration from BM by down-regulating CCR2 expression.
\end{abstract}

Yali Jiang and Yuanyuan Wang contributed equally to this work.

Electronic supplementary material The online version of this article (https://doi.org/10.1007/s13238-018-0527-6) contains supplementary material, which is available to authorized users.
Finally, we found that myeloid-specific Notch activation aggravated renal fibrosis, which was mediated by CCR2 $^{+}$macrophages infiltration. In summary, our data have unveiled that myeloid-specific targeting of Notch could ameliorate renal fibrosis by regulating BM-derived macrophages recruitment and activation, providing a novel strategy for intervention of this disease.

KEYWORDS renal fibrosis, Notch signaling, macrophages, heterogeneity, EMT

\section{INTRODUCTION}

Renal fibrosis is a common pathological process in endstage chronic kidney diseases (CKD) including chronic glomerulonephritis, diabetic nephropathy, and ureteral obstruction (Vernon et al., 2010). The dominant feature of renal fibrosis is excessive deposition of extracellular matrix (ECM) in kidney interstitium, which is deteriorated by inflammation, myofibroblast accumulation, and tubular atrophy (Cochrane et al., 2005). So far, no specific therapy has been established to cure renal fibrosis, due to poorly defined cellular and molecular mechanisms of this disease.

Macrophages play pivotal roles in renal fibrosis (Vernon et al., 2010; Wang and Harris, 2011), as depletion of macrophages ameliorates murine renal fibrosis induced by unilateral ureteral obstruction (UUO) (Kitamoto et al., 2009), suggesting that macrophages could serve as a therapeutic target. However, extensive observations have suggested that some macrophages exhibit pro-fibrotic activities, while 
others play an anti-fibrotic role (Kluth et al., 2004; Wang et al., 2007; Henderson et al., 2008). This controversial is reminiscent of recent findings unveiling the ontogenic heterogeneity and functional plasticity of macrophages (Wynn and Vannella, 2016). Macrophages in adult tissues are constituted by resident macrophages established during embryogenesis, and monocytes-derived macrophages recruited from bone marrow (BM). Most resident macrophages persist in tissues life-long and replenish themselves through self-renewal. Monocytes, on the other hand, are mobilized after tissue injury depending on chemotaxis signaling, and recruited to the inflammatory sites, where they differentiate into macrophages. Macrophages in tissues are activated and polarized into different subpopulations depending on local immunological milieu (Ginhoux and Guilliams, 2016). In response to IFN- $\gamma$ and/or LPS, macrophages adopt the $\mathrm{M} 1$ activation and produce TNF- $\alpha, \mathrm{IL}-1 \beta$, and NO, leading to enhanced inflammation, Th1-biased immune response and exacerbated tissue injury. In contrast, upon treatment with IL-4/IL-13, macrophages are polarized into M2 activation characterized by the up-regulated expression of IL-10, TGF- $\beta$, ARG1, and YM1. M2 macrophages exhibit anti-inflammatory activities and Th2 response, which promote tissue repair and remodeling (Vernon et al., 2010; Wynn and Vannella, 2016). In renal fibrosis, it has been reported that M1 macrophages exert pro-fibrotic role whereas M2 macrophages are anti-fibrotic (Nishida et al., 2005; Wang and Harris, 2011). Nevertheless, M2 macrophages express high level of TGF- $\beta$, a pro-fibrotic cytokine which promotes ECM deposition and epithelial-tomesenchymal transition (EMT), leading to renal fibrogenesis (Miyajima et al., 2000). Unveiling the role of these different populations of macrophages in renal fibrosis is a prerequisite of macrophages-targeted therapy.

A number of signaling pathways regulate macrophage recruitment, activation and proliferation. The CCL2-CCR2 signaling is essential for the emigration of monocytes from BM into blood stream (Kitagawa et al., 2004; Seki et al., 2009). M-CSF/C-fms and GM-CSF/CSF2R pathways support the proliferation of resident macrophages in response to altered microenvironment (Le Meur et al., 2002; Hashimoto et al., 2013). In addition, several cell-intrinsic pathways, such as p38/MAPK-, c-Jun/JNK-, and ERK-mediated signaling, have been implicated in macrophages recruitment and activation (Han et al., 2008; Ma et al., 2009). The Notch pathway constitutes contact-mediated cell-cell signaling (ArtavanisTsakonas et al., 1999). Notch activation is initiated by $\mathrm{Y}$ secretase-dependent cleavages of Notch receptors, liberating the Notch intracellular domain (NIC) that translocates into nuclei to associate with the recombination signal binding protein-JK (RBP-J). This event leads to the transactivation of downstream genes that are responsible for cell proliferation and differentiation (Chen and Al-Awqati, 2005; Hu and Phan, 2016). Notch signaling is critically involved in macrophage differentiation and activation in different disease models (Wang et al., 2010; Zhang et al., 2010; Xu et al., 2012;
Franklin et al., 2014; Zhao et al., 2016). Recently, our data have shown that disruption of RBP-J in macrophages ameliorates hepatic fibrosis by attenuating inflammation through cylindromatosis (CYLD) in mice (He et al., 2015). However, owing to the complicated roles of Notch in macrophage differentiation and activation, it is valuable to determine the role of Notch signaling in macrophages during renal fibrogenesis. In this study, we show that myeloid-specific Notch signaling significantly modulate renal fibrogenesis as in liver. However, in contrast to hepatic fibrosis, Notch signal regulates macrophages with distinct mechanisms in renal fibrosis, namely the CCR2-mediated monocyte recruitment and local macrophage activation. Collectively, our study indicated that targeting Notch signal in macrophages may be a new therapeutic strategy for kidney fibrosis.

\section{RESULTS}

Myeloid-specific RBP-J deficiency attenuated UUOinduced renal fibrosis

We first established UUO-induced renal fibrosis in normal mice. Masson's staining and hematoxylin and eosin (H\&E) staining showed that renal fibrosis was induced 1 week or 2 weeks after UUO (Fig. S1A and S1B). Meanwhile, immunofluorescence staining indicated that the infiltration of macrophages increased significantly in obstructed kidney as compared with the contralateral kidney (Fig. S1C), consistent with previous reports (Kitamoto et al., 2009; Vernon et al., 2010; Wang and Harris, 2011).

Notch signaling is critically involved in macrophage activation (Wang et al., 2010; Zhang et al., 2010; Xu et al., 2012; He et al., 2015; Zhao et al., 2016). To determine the role of Notch signaling in macrophages during renal fibrosis, $R B P$ $J$-floxed mice were crossed with $L y z 2-C r e$ transgenic mice to obtain macrophage-specific RBP-J knockout (Lyz2-Crel $\left.R B P-J^{f / f}, R B P-J \mathrm{cKO}\right)$ and control (Lyz2-Cre/RBP-J $J^{+/ f}$, Ctrl) mice according to our previous study (He et al., 2015). Quantitative (q) PCR using genomic DNA from sorted kidney macrophages showed that the efficiency of RBP-J deletion was almost complete in the RBP-J cKO mice (Fig. S2A and $\mathrm{S} 2 \mathrm{~B})$. Myeloid development was not altered apparently in the $R B P-J$ cKO mice (data not shown) (He et al., 2015).

Renal fibrosis was induced by UUO in the RBP-J cKO and control mice. Masson's staining and Sirius Red staining on day 14 after the operation indicated that renal fibrosis was markedly reduced in the $R B P-J$ cKO mice: the interstitial collagen area in the fibrotic kidney of the RBP-J cKO mice was about half of that in the control (Fig. 1A and 1B). Consistently, the expression of $\alpha$-smooth muscle actin ( $\alpha-S M A)$, a marker of myofibroblasts (Lin et al., 2009), decreased remarkably in the fibrotic kidney of $R B P-J$ cKO mice as determined using immunohischemistry staining (Fig. 1A lower panel and $1 \mathrm{C}$ ). We then examined the mRNA level of a-SMA and collagen I using qRT-PCR, and found that the expression of both molecules was significantly reduced in 
A Ctrl

A
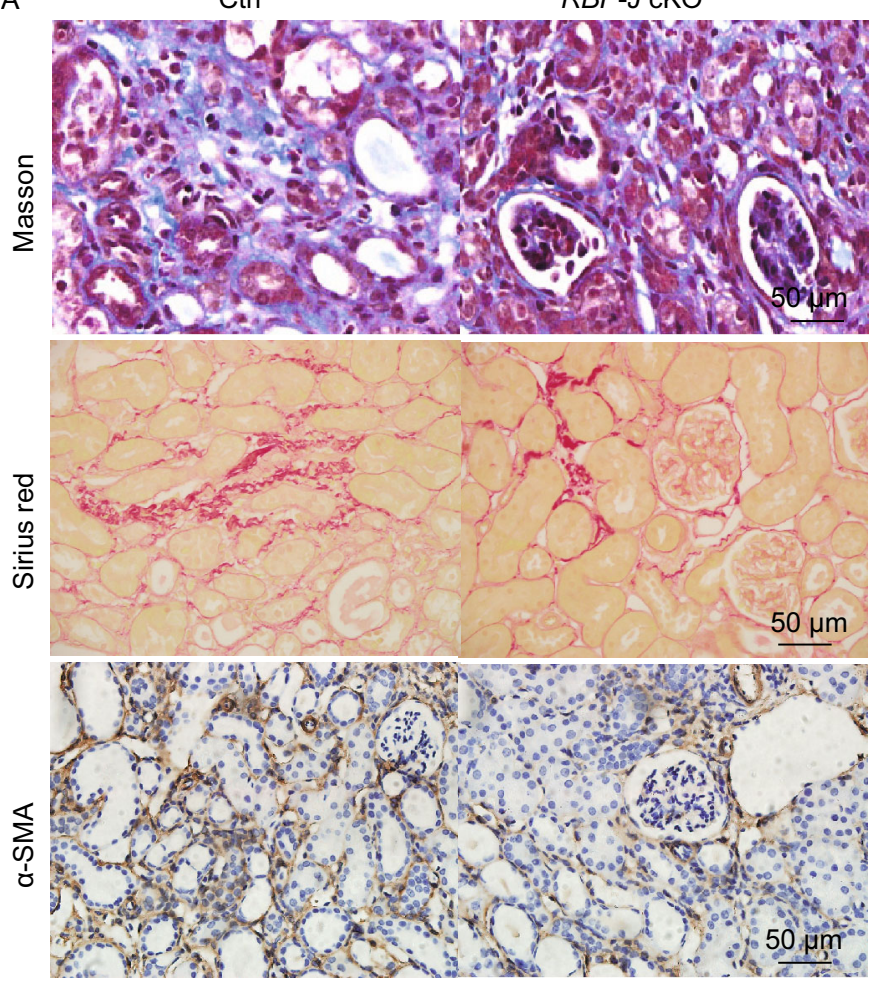
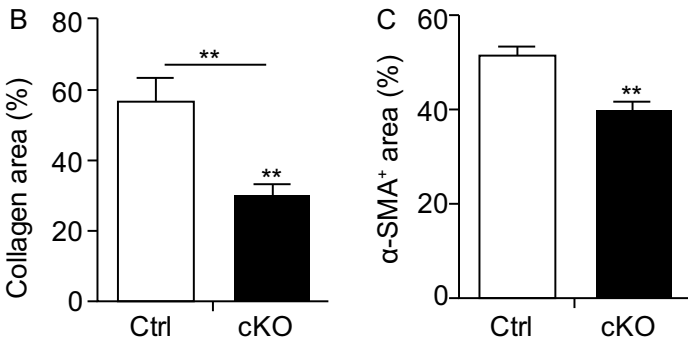

D

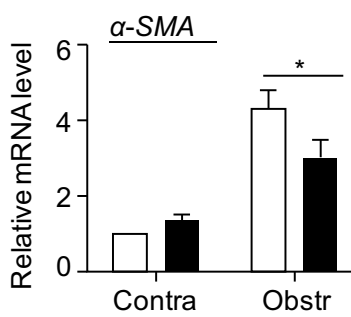

$\square$ Ctrl

- $\mathrm{cKO}$

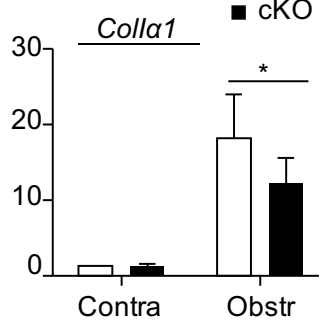

Figure 1. Myeloid-specific RBP-J deficiency attenuated UUO-induced renal fibrosis. (A) The RBP-J cKO and control mice were subjected to UUO. Masson and Sirius red staining, and anti- $\alpha$-SMA immunohistochemistry staining of kidney sections were performed 2 weeks after the operation. (B) The collagen-positive areas in (A) were quantified and compared. (C) The $\alpha$-SMA-positive areas in (A) were quantified in the RBP-J cKO and control mice and compared. (D) The kidneys from the RBP-J cKO and control mice were analyzed for the relative mRNA expression of $\alpha-S M A$ and Colla1 2 weeks after UUO using RT-PCR. Bars $=$ mean $\pm \mathrm{SD}, n=6$. *, $P<0.05,{ }^{* *}, P<0.01$.

the fibrotic kidney of RBP-J cKO mice as compared with the control (Fig. 1D). These results indicated that myeloidspecific disruption of RBP-J resulted in attenuated renal fibrosis in mice.

Inhibited expression of pro-fibrogenic factors and reduced EMT in the kidney of RBP-J cKO mice after UUO

TGF- $\beta$ is one of the major cytokines promoting renal fibrogenesis through EMT (Zavadil and Böttinger, 2005; Böttinger 2007; Kalluri and Weinberg, 2009). Therefore, we examined TGF- $\beta$ expression in the kidney of the RBP-J cKO and control mice subjected to UUO using qRT-PCR and ELISA. The results showed that TGF- $\beta$ was markedly down-regulated in the fibrotic kidney of the RBP-J cKO mice as compared with the control (Fig. 2A and 2B). Then, we evaluated EMT in the fibrotic kidneys of the RBP-J cKO and control mice by determining the expression of the EMT-related markers using qRT-PCR and Western blotting. The result showed that, compared with the control, the epithelial marker E-cadherin was higher whereas the mesenchymal markers Vimentin and $\mathrm{N}$-cadherin was lower in the fibrotic kidney of the RBP-J cKO mice as compared with that of the control (Fig. 2C and 2D). The expression of Snail, a transcription factor driving EMT, was also lower in the fibrotic kidney of the RBP-J cKO mice (Fig. 2C). Therefore, myeloidspecific deletion of $R B P-J$ ameliorated renal fibrosis through, at least in part, the attenuated TGF- $\beta$ expression and EMT in kidney.

To further verify whether RBP-J deficiency influenced the ability of macrophages to induce EMT, we cultured primary proximal tubular epithelial cells (PTEpiC) of wild-type mice with the conditional medium (CM) collected from cultured kidney macrophages that were sorted from fibrotic kidney of the RBP-J cKO or control mice for $24 \mathrm{~h}$. The phenotype and purity of sorted kidney macrophages was shown in Supplementary Figure 3A. The morphology of the tubule cells cultured with the control $\mathrm{CM}$ became fusiform, similar to activated fibroblasts, whereas the tubule cells cultured with the RBP-J cKO macrophage-derived CM kept cobblestonelike appearance of normal tubule cells (Fig. S3B). After collecting co-cultured PTEpiC, the mRNA expression of EMTand fibrosis-related markers was examined using qRT-PCR. 


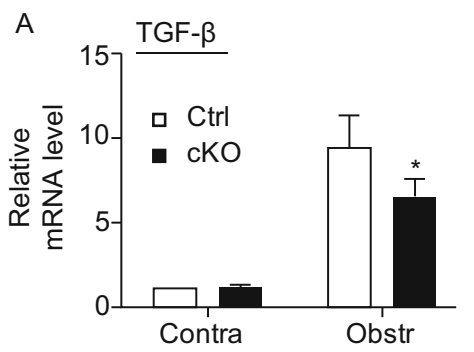

B

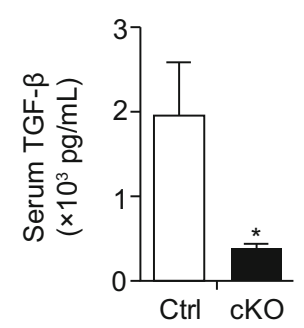

D

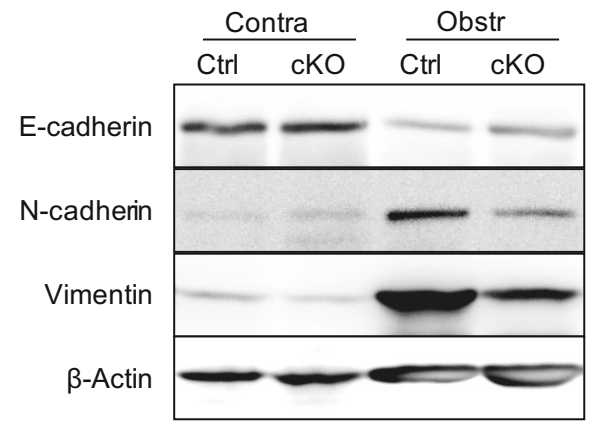

C

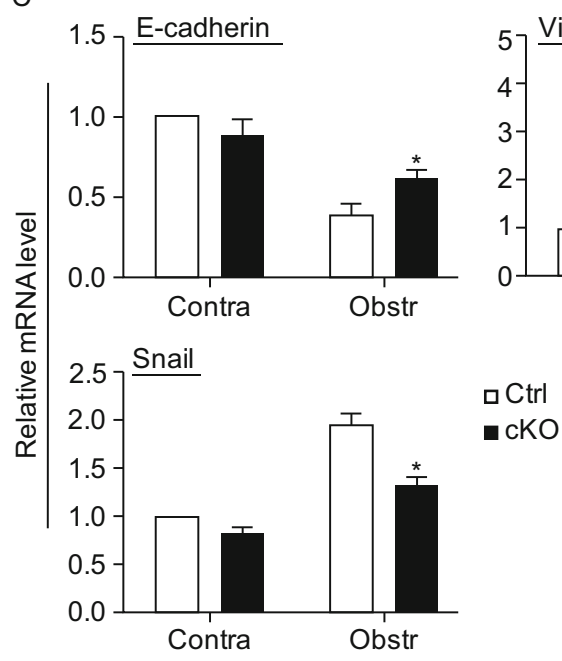

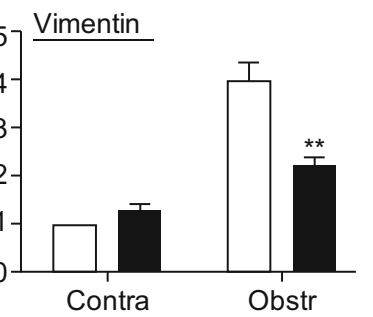

Ctrl

E
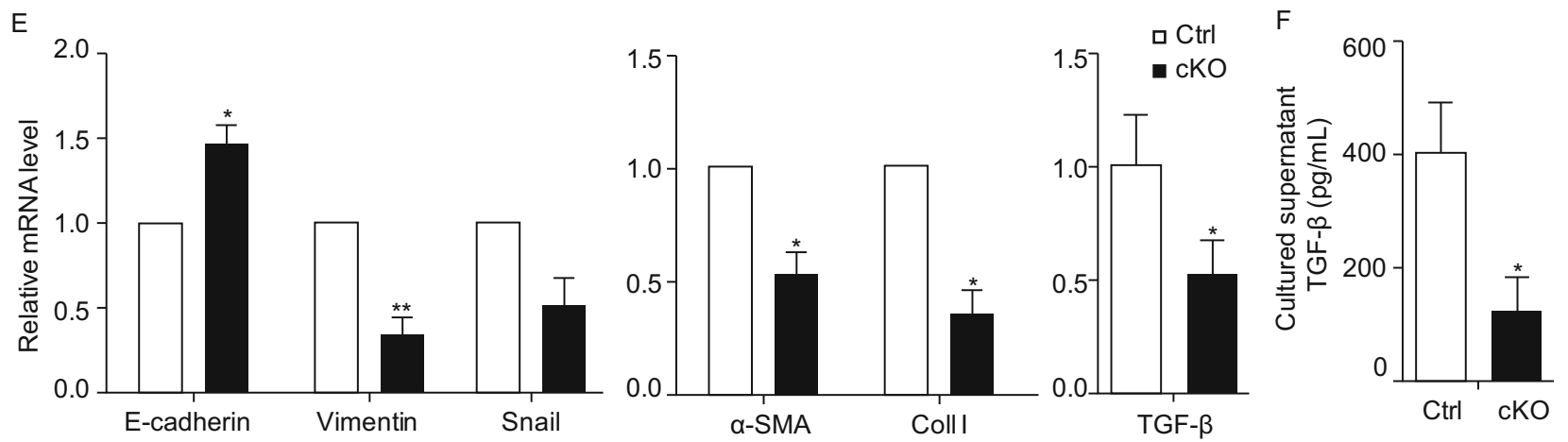

Figure 2. Decreased expression of TGF- $\beta$ and EMT in the kidney of myeloid-specific RBP-J deficient mice after UUO. (A) Relative mRNA expression of TGF- $\beta$ in kidney from the RBP-J cKO and control mice was determined 2 weeks after UUO using RT-PCR $(n=6)$. (B) The level of TGF- $\beta$ in serum of the RBP-J cKO and control mice was determined with ELISA $(n=6)$. (C) Relative mRNA expression of EMT-related markers in the kidney was determined by RT-PCR $(n=6)$. (D) The protein level of EMT-related markers in the kidney extracts was detected by Western blotting, and quantitatively compared between groups $(n=6)$. (E) Primary proximal tubular epithelial cells were isolated from normal mice, and cultured for $24 \mathrm{~h}$ in the presence of the conditional medium (CM) from kidney macrophages from the RBP-J cKO or control mice suffering from UUO for 2 weeks. And then the co-cultured tubular epithelial cells were harvested for detecting mRNA expression of EMT- and fibrosis-related markers using qRT-PCR $(n=3)$. (F) the protein level of TGF- $\beta$ was detected in conditional medium from cultured kidney macrophages of fibrotic kidney of $R B P-J$ cKO and control mice by $\operatorname{ELISA}(n=3)$. Bars $=$ mean \pm SD. ${ }^{*}, P<0.05,{ }^{* *}, P<0.01,{ }^{* * *}, P<0.001$.

The result showed that the mRNA level of E-cadherin increased while that of Vimentin, Snail, a-SMA, collagen I and TGF- $\beta$ decreased in PTEpiC cultured with the RBP-J cKO macrophages-derived $\mathrm{CM}$ compared with the control
$\mathrm{CM}$ (Fig. 2E). Meanwhile, the expression of TGF- $\beta$ in cultured supernatant of RBP-J cKO macrophages was decreased significantly as compared with the control (Fig. 2F). Taken together, these results suggested that RBP- 
A

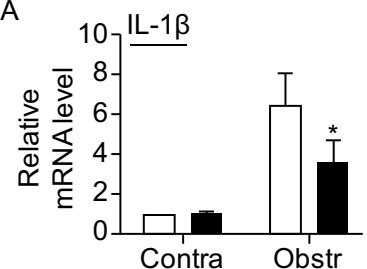

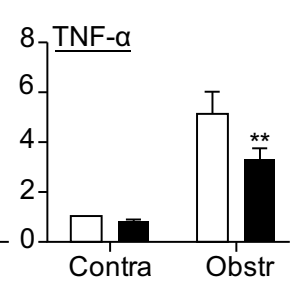

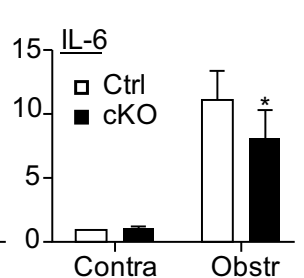

C Contra

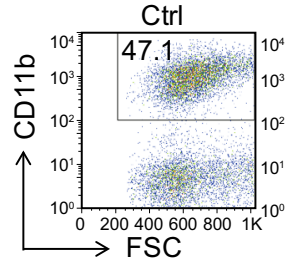

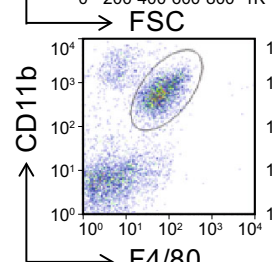
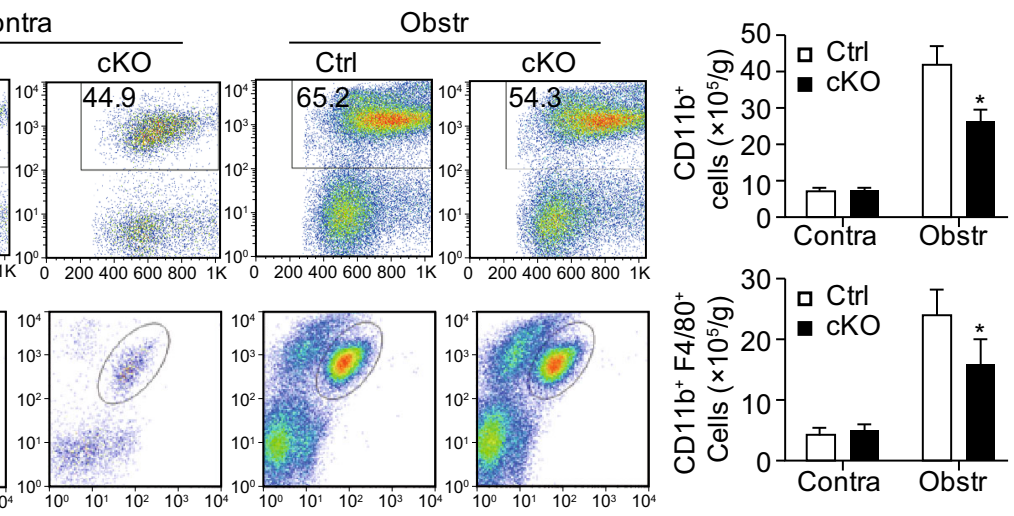

$\mathrm{E}$
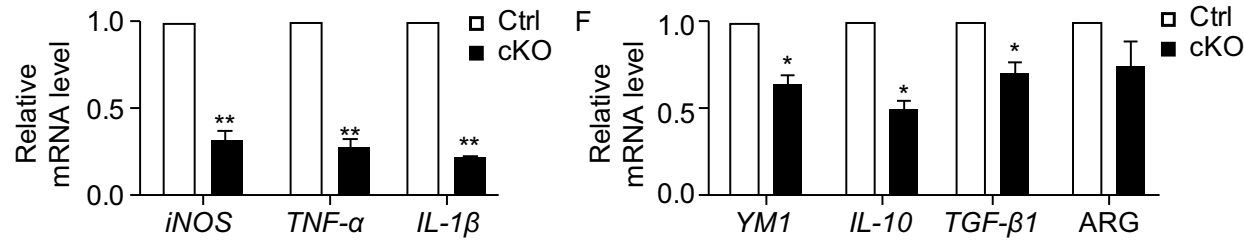

Figure 3. Ameliorated inflammation and macrophage infiltration and activation in fibrotic kidney of myeloid-specific RBP-J deficient mice after UUO. (A) RBP-J cKO and control mice were subjected to UUO. The mRNA expression of $I L-1 \beta, T N F-\alpha$ and $I L-6$ in the kidney was determined 2 weeks after UUO by qRT-PCR. (B) The protein level of TNF- $\alpha$ was detected by ELISA in serum of $R B P-J$ CKO and control mice in (a). (C) Single cell suspensions were prepared from obstructed and contralateral kidney of $R B P-J$ cKO and control mice respectively, and then performed FACS analysis. CD11 $\mathrm{b}^{+}$cells (myeloid cells) and $\mathrm{CD} 11 \mathrm{~b}^{+} \mathrm{F} 4 / 80^{+}$cells (macrophages) were quantitatively compared. (D) Kidney sections in (a) were stained with anti-F4/80 antibody using immunofluorescence staining, and the $\mathrm{F} 4 / 80^{+}$macrophages were counted and compared. (E and F) Single cell suspensions were prepared from the kidneys 2 weeks after the UUO, and macrophages $\left(C D 11 \mathrm{~b}^{+} \mathrm{F} 4 / 80^{+}\right)$were sorted and then cultured in vitro for $24 \mathrm{~h}$. The mRNA expression of M1 (E) and M2 (F) macrophage activation markers was determined using qRT-PCR. Bars $=$ mean $\pm \mathrm{SD}, n=6$. *, $P<0.05,{ }^{* *}, P<0.01$.

$\mathrm{J}$ deficient macrophages exhibited reduced capacity of inducing EMT of tubular epithelial cells.

Decreased macrophage infiltration and activation in the kidney of RBP-J cKO mice upon UUO

H\&E staining showed reduced infiltration of inflammatory cells in the interstitial regions of the fibrotic kidney of the $R B P-J$ cKO mice after UUO, suggesting a compromised inflammation (Fig. S4A). Consistently, the level of inflammatory cytokines including TNF- $\alpha$, IL-1 $1 \beta$, and IL-6 decreased significantly in the fibrotic kidney of RBP-J cKO mice as compared with the control (Fig. $3 A$ and $3 B$ ).

Next we analyzed myeloid populations in the fibrotic kidney of the RBP-J cKO and control mice using FACS (Lin et al., 2009). The total number of infiltrated granulocytes $\left(\mathrm{CD} 11 \mathrm{~b}^{+} \mathrm{Ly}_{6 \mathrm{G}}{ }^{\mathrm{hi}}\right)$ in the fibrotic kidney of the RBP-J cKO mice was comparable with that of the control (Fig. S4B). Furthermore, we detected the significantly reduced $C D 11 b^{+}$ myeloid cells and $\mathrm{CD} 11 \mathrm{~b}^{+} \mathrm{F} 4 / 80^{+}$macrophages in the fibrotic kidney of the RBP-J cKO mice than in the control (Fig. 3C). Immunofluorescence staining with anti-F4/80 also showed that $\mathrm{F} 4 / 80^{+}$macrophages decreased obviously in the fibrotic kidney of the RBP-J CKO mice (Fig. 3D). These data suggested that attenuated inflammatory response in the fibrotic kidney of RBP-J cKO mice likely resulted from reduced inflammatory macrophages infiltration.

To further evaluate the role of Notch signaling in regulating macrophages in renal fibrosis, CD $11 \mathrm{~b}^{+} \mathrm{F} 4 / 80^{+}$macrophages were sorted from the fibrotic kidney of the RBP-J 
cKO and control mice, and the expression of macrophage activation markers was determined using qRT-PCR. The results showed that the expression of both of the $M 1$ markers iNOS, TNF- $\alpha, I L-1 \beta$, and the $M 2$ markers $Y M 1, I L-10$ and $T G F-\beta$ was reduced obviously in kidney macrophages from the RBP-J cKO mice during renal fibrosis (Fig. $3 \mathrm{E}$ and $3 \mathrm{~F}$ ), indicating that macrophages activation was attenuated in the fibrotic kidney of macrophage-specific RBP-J deficient mice. Meanwhile, the reduced TGF- $\beta$ expression in RBP-J deficient macrophages may responsible for decreased EMT in fibrotic kidney of RBP-J cKO mice as shown in Fig. 2.

\section{Macrophage-specific RBP-J cKO did not affect macrophage proliferation}

In situ proliferation stimulated by local cytokines such as CSF-1 or IL-4 plays an important role in maintaining macrophage homeostasis in tissues (Le Meur et al., 2002; Hashimoto et al., 2013). We then asked whether Notch signaling could influence renal fibrogenesis by regulating macrophage proliferation in kidney. The RBP-J cKO and control mice were injected with BrdU and subjected to UUO. FACS analysis showed that while the percentage of proliferating macrophages was significantly higher in the fibrotic kidney as compared with the contralateral kidney, the absolute number of $\mathrm{BrdU}^{+} \mathrm{F} 4 / 80^{+}$macrophages in the fibrotic kidneys of RBP-J cKO mice was comparable with that of the control (Fig. 4A and 4B). This suggested that Notch signaling did not affect local macrophage proliferation in UUO-induced renal fibrosis.

\section{Notch signaling regulated renal fibrosis mainly through monocytes-derived macrophages}

Tissue resident macrophages have been implicated in tissue injury and repair (Yamasaki et al., 2014; Zigmond et al., 2014; Stamatiades et al., 2016). In order to address the role of different origins of kidney macrophages, the RBP-J cKO and control mice were injected with liposome-encapsulated clodronate to deplete embryonic-derived kidney resident macrophages before UUO according to published protocol (Kitamoto et al., 2009), and then mice were subjected to UUO for 7 days. FACS analyses showed that the CD11 ${ }^{+} \mathrm{F} 4 /$ $80^{+} \mathrm{CX} 3 \mathrm{CR} 1^{+}$resident macrophages were almost completely depleted by clodronate-liposomes (CLs) treatment (Fig. 5A). In contrast, although the CD11 ${ }^{+} \mathrm{F} 4 / 80^{+} \mathrm{CCR} 2^{+}$ monocytes-derived macrophages were also affected, this population was still less in RBP-J cKO mice than that in control mice irrespective of tissue resident macrophage depletion (Fig. 5B). Meanwhile, upon CLs treatment, the infiltrated inflammatory cells and the degree of renal fibrosis in RBP-J cKO mice and control mice did not alter obviously compared with those after liposome treatment (PLs), as shown by H\&E staining (Fig. 5C left panel and 5D) and Masson's staining (Fig. $5 \mathrm{C}$ right panel and 5E). These results indicated that Notch signaling regulated renal fibrosis
A

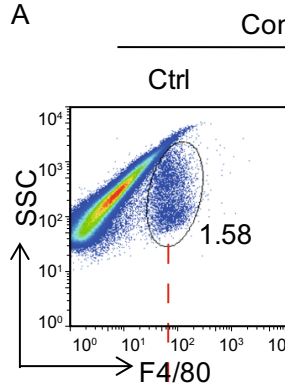

Contra

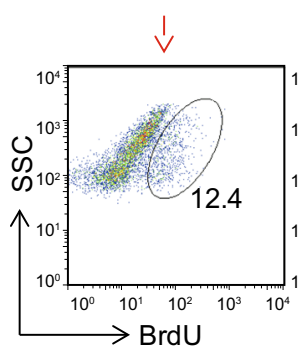

cKO

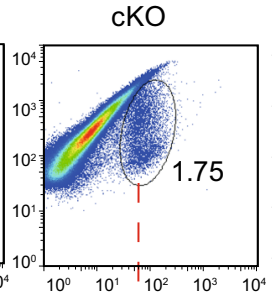
75
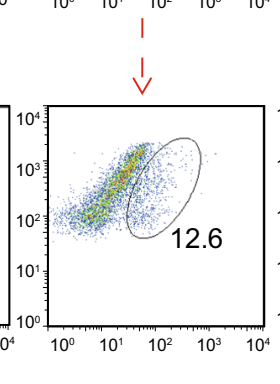

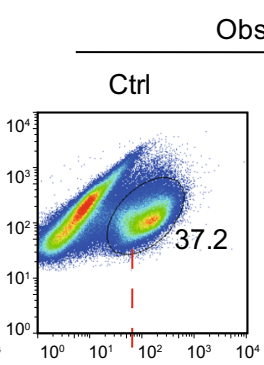

Obstr
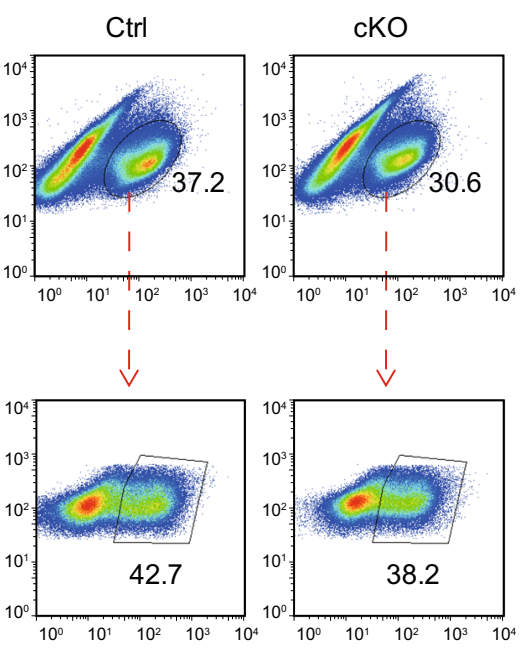

Figure 4. No significant difference of macrophage proliferation in kidney between RBP-J cKO and control mice after UUO. (A) RBP-J cKO and control mice were subjected to UUO and injected i.p. with BrdU. The BrdU-labeled proliferating macrophages were determined by FACS 7 days after the UUO. (B) F4/80 $\mathrm{BrdU}^{+}$cells in (A) were quantitatively compared. Bars $=$mean $\pm \mathrm{SD}, n=3$. ns, not significant. 
A

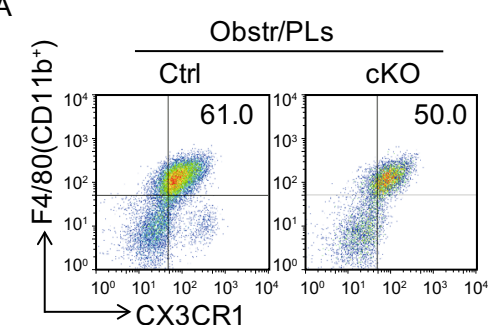

B
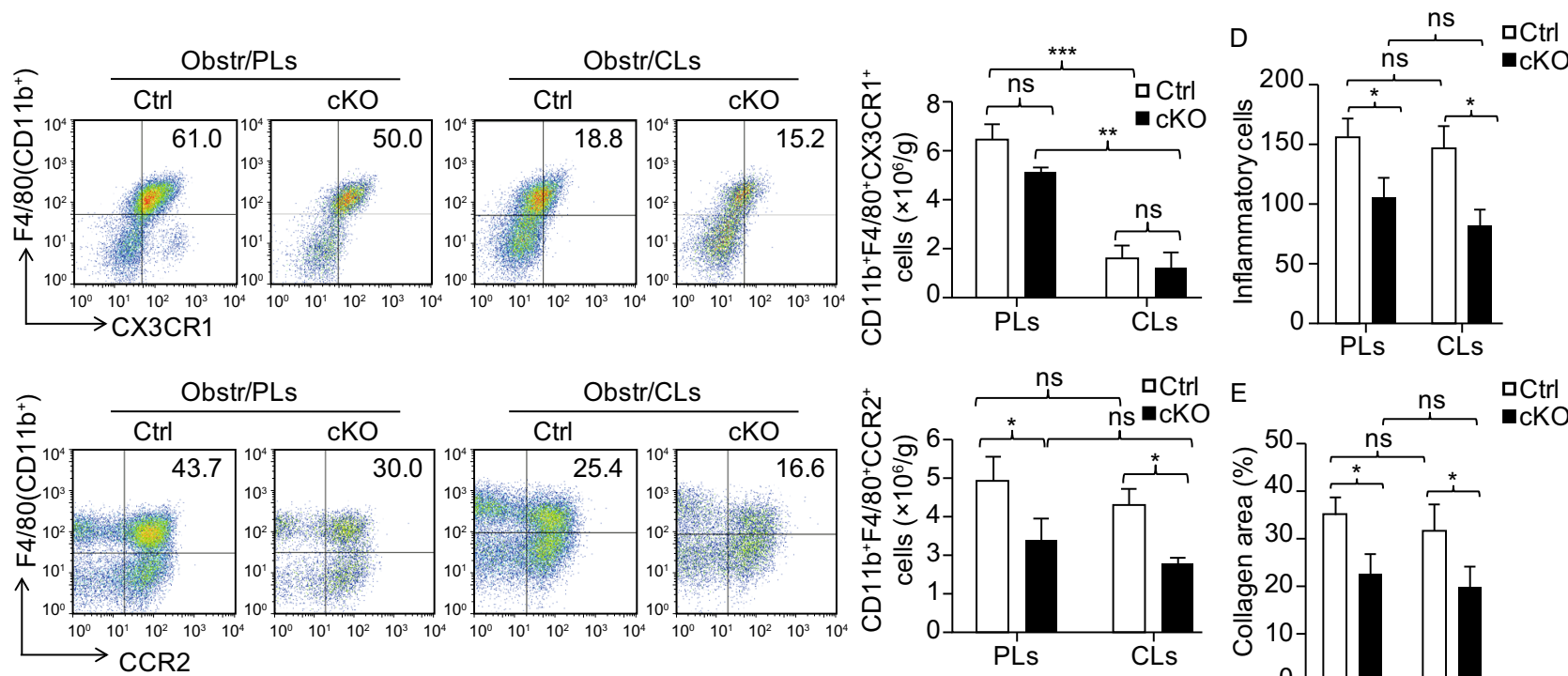

C

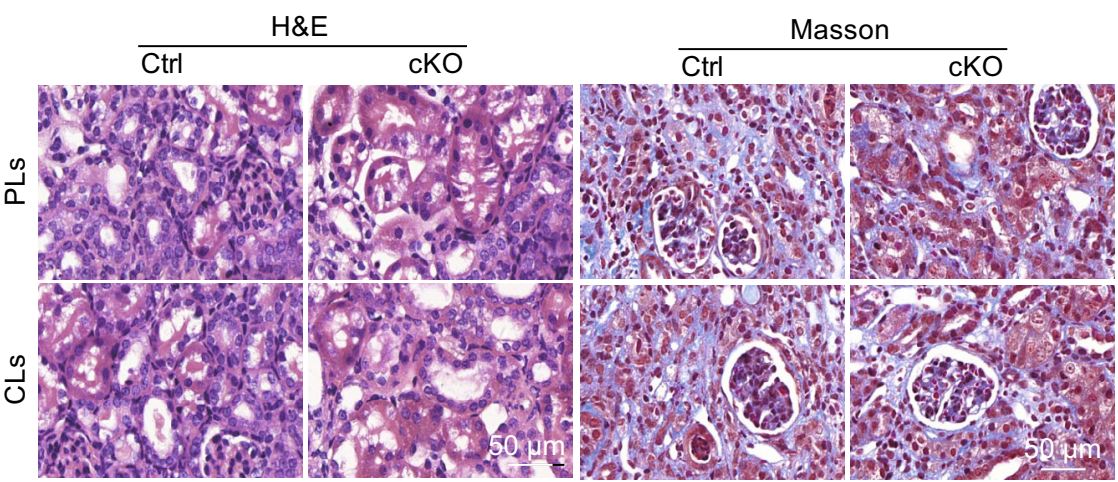

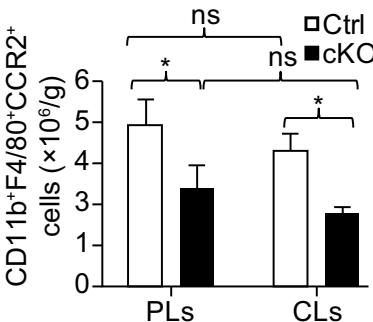

CLs

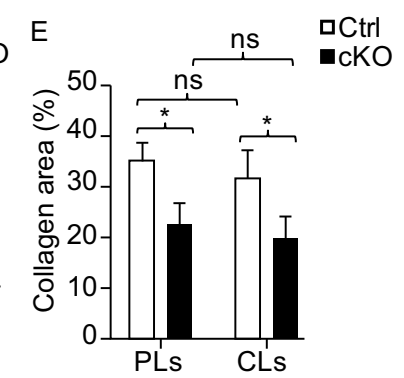

Figure 5. RBP-J cKO attenuated UUO-induced renal fibrosis independent on kidney resident macrophages. $R B P-J \mathrm{cKO}$ and control mice were injected i.v. With liposome-encapsulated clodronate (CLs) or control liposome (PLs) three times before UUO. Kidney macrophages were analyzed by FACS 7 days after UUO, and the number of CD $11 \mathrm{~b}^{+} \mathrm{F} 4 / 80^{+} \mathrm{CX} 3 \mathrm{CR} 1^{+}$resident macrophages (A) and $C D 11 b^{+} F 4 / 80^{+} C C R 2^{+}$BM-derived macrophages (B) were quantified and compared. (C) H\&E staining (left panel) and Masson's staining (right panel) was performed using kidney sections. (D) The infiltrating inflammatory cells were counted and compared using $\mathrm{H} \& \mathrm{E}$ staining sections in (C). (E) The collagen-positive areas were quantified and compared using Masson's staining sections in (C). Bars $=$ mean $\pm \mathrm{SD}, n=3 .{ }^{*}, P<0.05$, ns, not significant.

likely through the monocytes-derived macrophages rather than the kidney resident macrophages.

Myeloid-specific RBP-J deficiency led to reduced recruitment of $\mathrm{CCR}^{+}$monocytes from BM after UUO

Because RBP-J deficiency resulted in reduced inflammatory macrophage infiltration in fibrotic kidney, we next investigated the expression of CCR2, a critical chemokine receptor involved in monocyte migration (Kitagawa et al., 2004; Seki et al., 2009), in kidney macrophages of the RBP-J cKO and control mice by FACS. Both the number of $C D 11 b^{+} F 4 /$ $80^{+} \mathrm{CCR} 2^{+}$macrophages and the mean fluorescence intensity (MFI) of $\mathrm{CCR}^{+}$macrophages decreased significantly in the fibrotic kidneys of the RBP-J cKO mice (Fig. 6A). FACS analysis also indicated that the number of $C D 11 b^{+} C C R 2^{+}$ monocytes increased in the BM but decreased in the spleen of the RBP-J cKO mice during renal fibrosis (Fig. 6B-E). Moreover, the mRNA level of CCL2 in the fibrotic kidney of $R B P-J$ cKO mice was also reduced significantly, which might further contribute to the reduced recruitment of $\mathrm{CCR}^{+}$ monocytes into the fibrotic kidney in the RBP-J cKO mice (Fig. 6F). These results suggested that the recruitment of inflammatory monocytes from BM, which is mediated by CCL2-CCR2 chemotaxis, was damaged in the RBP-J cKO mice undergoing UUO-induced renal fibrosis, likely leading to reduced infiltration of inflammatory macrophages.

To further verify that Notch signaling regulated macrophage infiltration through CCL2-CCR2 chemotaxis, we cocultured RBP-J deficient or control macrophages with HEK293 cells overexpressing CCL2 using a transwell system. The result showed that significantly less RBP-J 
A
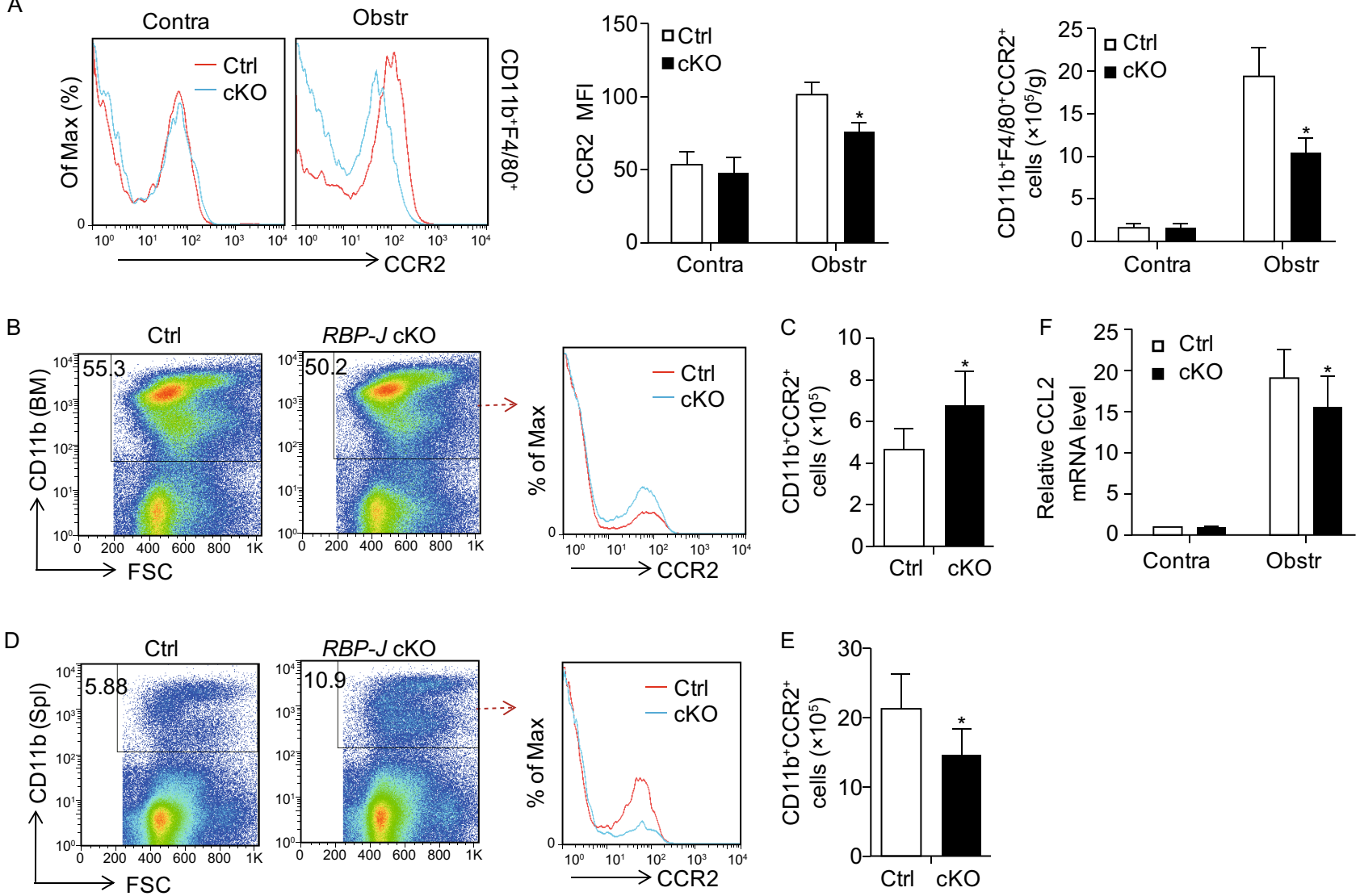

G
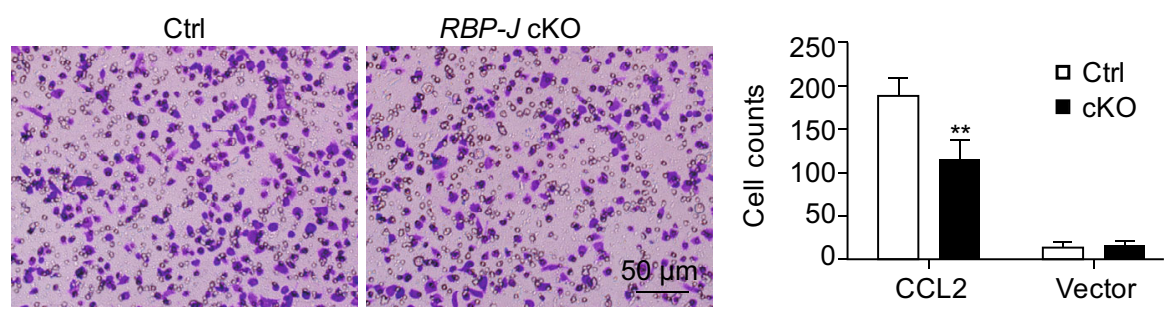

$\mathrm{H}$
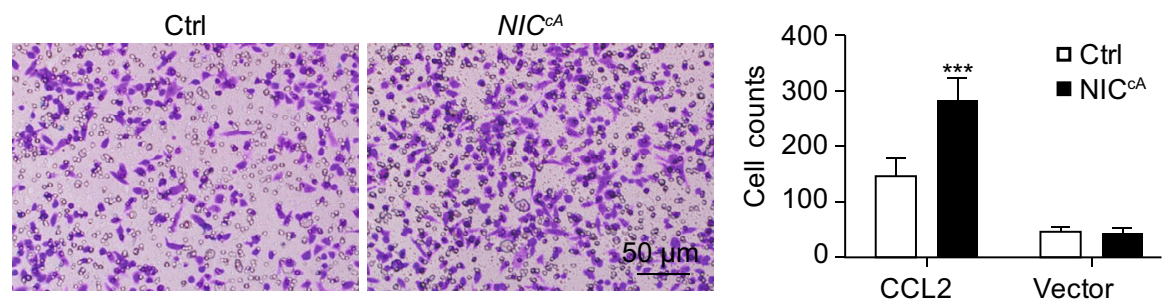

deficient macrophages migrated towards CCL2-expressing cells as compared with the control (Fig. 6G). In contrast, when macrophages derived from myeloid-specific NIC transgenic mice (Zhao et al., 2016) (NIC ${ }^{C A}$, see below) were co-cultured with the CCL2-expressing HEK293 cells using transwell system, we found that more macrophages with activated Notch signaling migrated towards CCL2-expressing cells as compared with the control (Fig. $6 \mathrm{H})$. These data suggested that Notch signaling regulated macrophage infiltration in fibrotic kidney most likely through the CCL2CCR2 chemotaxis.

Although the MFI of $\mathrm{CCR}^{+}$macrophages was decreased significantly in the fibrotic kidney of the RBP-J cKO mice (Fig. 6A), the mRNA level of CCR2 decreased slightly in RBP-J cKO macrophages (Fig. S4C), suggesting that Notch signaling might regulate CCR2 expression on transcription or post-transcription level. Therefore, we isolated monocytes from the BM of the $N I C^{C A}$ mice, and performed a chromatin 
Figure 6. Myeloid RBP-J deficiency led to the reduced recruitment of $\mathrm{CCR2}^{+}$monocytes from BM after UUO through CCR2-CCL2 chemotaxis. (A) RBP-J CKO and control mice were subjected to UUO. The expression of CCR2 in CD11 ${ }^{+} \mathrm{F} 4 /$ $80^{+}$macrophages from obstructed and contralateral kidneys was determined by FACS (right panel). The mean fluorescence intensity (MFI) of CCR2 expression (middle panel) and the number of $\mathrm{CCR}^{+}$inflammatory monocytes-derived macrophages were quantitatively compared (left panel) $(n=6)$. (B and C) BM from the RBP-J cKO and control mice was collected 2 weeks after the UUO, and the $\mathrm{CD}_{11} \mathrm{~b}^{+}$monocytes were analyzed by using FACS for the expression of CCR2 (B). The number of $\mathrm{CCR}^{+}$inflammatory monocytes was quantitatively compared (C) $(n=6)$. ( $D$ and E) Spleenocytes were collected from the RBP-J cKO and control mice 2 weeks after the UUO, and the $C D 11 b^{+}$monocytes were analyzed by FACS for the expression of CCR2 (D). The number of $\mathrm{CCR}^{+}$inflammatory monocytes was quantitatively compared $(\mathrm{E})(n=6)$. (F) The mRNA level of CCL2 was determined by qRT-PCR in the fibrotic kidney of the RBP-J cKO and control mice after UUO $(n=6)$. (G) BMDMs from the RBP-J cKO or control mice were plated into the upper chamber of 24-well transwell chambers. The lower chambers were pre-plated for $24 \mathrm{~h}$ with HEK293 cells transfected with CCL2-expressing or the control plasmid. Cell migration was allowed for $3.5 \mathrm{~h}$ at $37^{\circ} \mathrm{C}$, and the migrated cells were quantitatively compared $(n=3)$. $(\mathrm{H})$ BMDMs from the myeloid-specific NIC transgenic or control mice were tested for chemotaxis to CCL2 as in $(\mathrm{G})(n=3)$. Bars $=$ mean $\pm \mathrm{SD}$. ${ }^{*}, P<$ $0.05,{ }^{* *}, P<0.01,{ }^{* \star *}, P<0.001$.

immunoprecipitation (ChIP) assay with anti-RNA polymerase (Pol) II or anti-NIC antibody. The result showed that the binding of RNA Pol II to the CCR2 promoter intended to increase slightly in Notch-activated monocytes, but the occupation of NIC on the CCR2 promoter appeared not changed, suggesting that Notch signaling might activate the transcription of CCR2 in macrophages indirectly (Fig. S5) or regulate the expression of $C C R 2$ in post-transcription level that need to be further explored.

\section{Myeloid-specific Notch activation aggravated UUO- induced renal fibrosis}

So far, our data indicated that disruption of Notch signaling in macrophages ameliorated UUO-induced renal fibrosis by reducing macrophage infiltration and activation. We then set out to confirm the effect of Notch signaling in macrophages on renal fibrosis by using the myeloid-specific NIC transgenic mice (Zhao et al., 2016) (Fig. S6). The Lyz2-Crel STOP flox $-N I C\left(N I C^{C A}\right)$ and control mice ( $\left.L y z 2-C r e\right)$ mice were subjected to UUO. Masson's staining and Sirius Red staining and H\&E staining showed that activation of Notch signaling in macrophages aggravated renal fibrosis following collagen deposition, as well as accompanied by increased inflammatory cells infiltration in the kidney interstitium (Fig. 7A, 7B and 7D). Consistently, more $\alpha-S_{M A}^{+}$myofibroblasts occurred in Notch activated macrophages mice during renal fibrogenesis (Fig. 7A lower panel and 7C). Meanwhile, FACS analysis showed that the infiltration of the $C D 11 b^{+} F 4 / 80^{+}$ macrophages in the fibrotic kidneys of $N I C^{C A}$ mice increased as compared with the control (Fig. $7 \mathrm{E}$ and $7 \mathrm{~F}$ ), and the $\mathrm{CD}_{11 \mathrm{~b}^{+}}$myeloid cells also increased significantly (Fig. S7A). The number of $\mathrm{CD} 11 \mathrm{~b}^{+} \mathrm{F} 4 / 80^{+} \mathrm{CCR} 2^{+}$macrophages, CCR2 MFI in macrophages and the level of CCL2 all increased in the fibrotic kidney of $N I C^{C A}$ mice as compared with the control (Figs. 7G, 7H, S7B and S7C). These results suggested that, contrary to the myeloid-specific RBP-J cKO mice, myeloid-specific Notch activation aggravated UUOinduced renal fibrosis through promoting $\mathrm{CCR}^{+}$macrophages recruitment.

\section{DISCUSSION}

Renal fibrosis is characterized by excessive deposition of ECM, which is produced primarily by myofibroblasts. Despite multiple origins of myofibroblasts as revealed by recent studies (LeBleu et al., 2013; Falke et al., 2015), the critical roles of macrophages in the formation and activation of myofibroblasts in renal fibrogenesis have been consensually appreciated (Vernon et al., 2010; Nikolic-Paterson et al., 2014). Recently, BM-derived macrophages are found to undergo the transition into $\alpha$-SMA-positive collagen-producing cells, namely activated myofibroblasts, during tissue fibrosis, (Wang et al., 2016; Meng et al., 2016). In our study, the expression of $\alpha-S M A$ or collagen I decreased remarkably in the fibrotic kidney of RBP-J CKO mice as determined using immunohistochemistry staining or qRT-PCR (Fig. 1). Given that Notch signaling directly regulates $\alpha-S M A$ and collagen I transcription (Tang et al., 2008; Hu et al., 2014), we could not formally exclude that RBP-J deficiency in macrophages might directly result in decreased production of $\alpha$-SMA and collagen I derived from macrophages in fibrotic kidney. This possibility is currently under investigation in our laboratory.

Macrophages are activated and modulated by cell debris and molecules bearing the damage-associated molecular patterns (DAMPs) released by injured cells, and by cytokines present in the specific immuno-microenvironment during CKD (Williams et al., 2010). Differentially activated macrophages exert different even contradictory influences on renal fibrogenesis through secreting a wide spectrum of cytokines, growth factors, chemokines, and other inflammatory factors (Ginhoux and Guilliams, 2016; Wynn and Vannella, 2016). Notch signaling is critically involved in macrophage activation (Monsalve et al., 2009; Wang et al., 2010; Zhang et al., 2010; Xu et al., 2012; Zhao et al., 2016). We have recently demonstrated that disruption of Notch signaling by myeloidspecific RBP-J knockout attenuated liver fibrosis by compromising macrophage activation through the CYLD-NF-KB pathway (He et al., 2015). In this study, we extended these findings to UUO-induced renal fibrosis, and found that blockade of Notch signaling by myeloid-specific RBP-J 
A

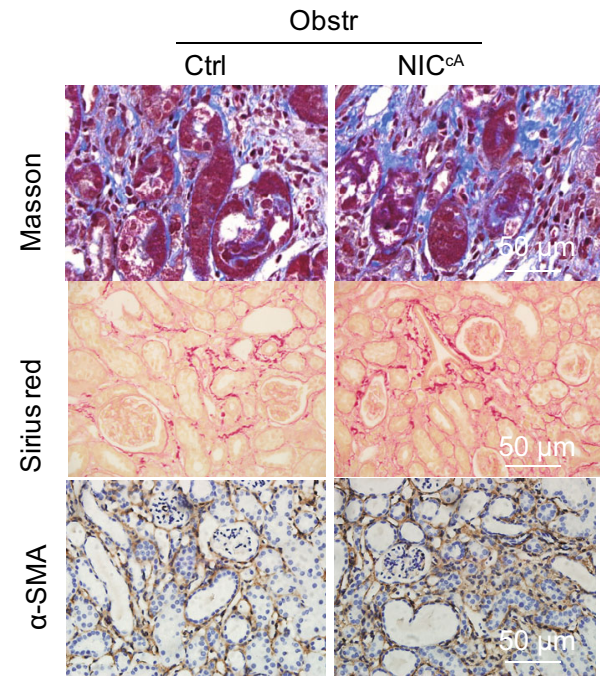

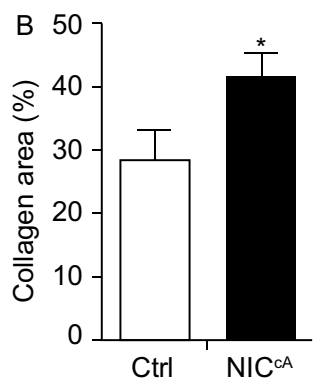
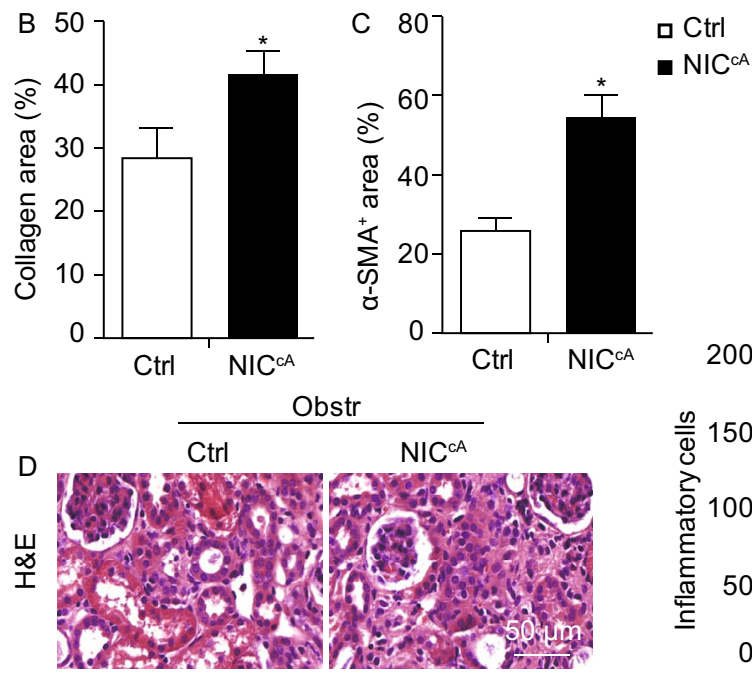

$\mathrm{F}$

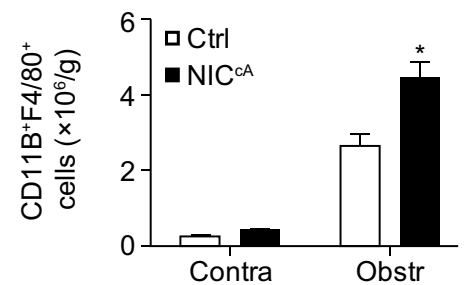

G

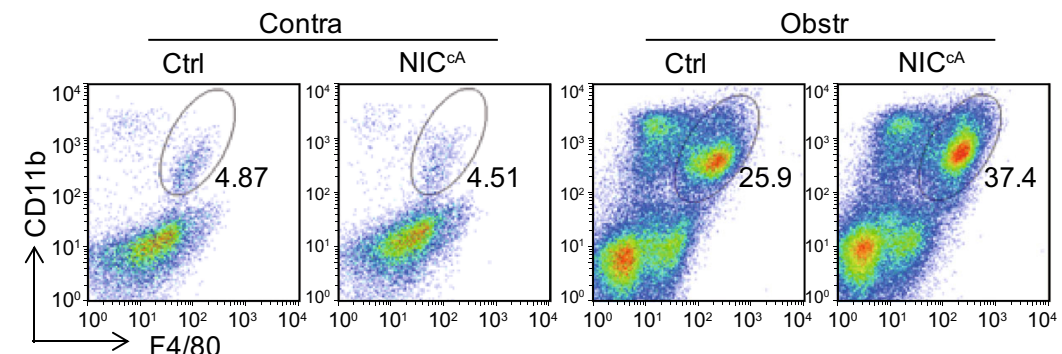

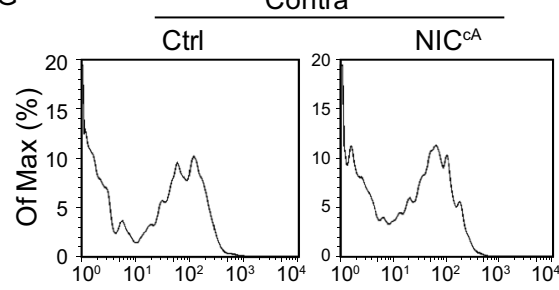

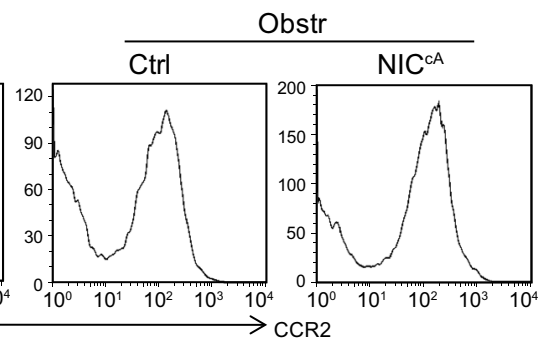

$\mathrm{H}$

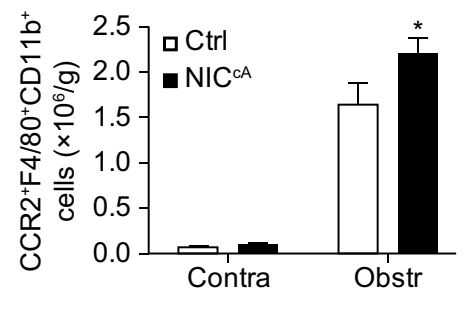

Figure 7. Myeloid-specific Notch activation aggravated renal fibrosis after UUO. Myeloid-specific NIC transgenic $\left(N I C^{C A}\right)$ and control mice were subjected to UUO for 2 weeks. (A-C) Masson (upper panels) and Sirius red staining (middle panel) and $\alpha-S M A$ staining (lower panels) in fibrotic kidney sections was performed (A). The collagen-positive areas (B) and $\alpha-S M A^{+}$areas $(C)$ were counted and compared. (D) H\&E staining of kidney sections were performed 2 weeks after the UUO, and the inflammatory cells in the kidney interstitial $(C)$ were quantified. ( $E$ and $F$ ) Single cell suspensions were prepared form the fibrotic kidneys. $C D 11 b^{+} F 4 / 80^{+}$ macrophages were determined by FACS $(E)$. The number of $C D 11 b^{+} F 4 / 80^{+}$macrophages in $(E)$ was quantitatively compared $(F)$. (G and $\mathrm{H}$ ) $\mathrm{CCR}^{+}$inflammatory macrophages in (E) were determined by FACS (G), and the number of $\mathrm{CD} 11 \mathrm{~b}^{+} \mathrm{F} 4 / 80^{+} \mathrm{CCR} 2^{+}$ macrophages was quantitatively compared $(\mathrm{H})$. Bars $=$ mean $\pm \mathrm{SD}, n=6$. ${ }^{*}, P<0.05$, ns, not significant.

knockout remarkably ameliorated renal fibrosis. In contrast, renal fibrosis was aggravated by using the myeloid-specific Notch activation mouse model. Therefore, we conclude that Notch activation is likely necessary for macrophage activation in tissue fibrogenesis. To inhibit Notch activation in macrophages may be a new strategy for fibrosis therapy, at least for liver and kidney fibrosis.

Tissue macrophages contain sub-populations with different ontogenies (Ginhoux and Guilliams, 2016; Wynn and Vannella, 2016). The homeostasis of macrophage repertoire in adults is maintained primarily by in situ tissue-resident macrophage proliferation and monocyte influx from BM, as well as programmed cell death involving apoptosis and/or necroptosis (Hashimoto et al., 2013; Yamasaki et al., 2014; Zigmond et al., 2014; Stamatiades et al., 2016). We thus questioned the cellular mechanism(s) by which Notch signaling regulated the macrophage repertoire during renal fibrosis. Our BrdU incorporation experiment indicated that local macrophage proliferation might not contributed to the Notch signaling-mediated regulation of macrophages in renal fibrogenesis. 
Moreover, the depletion of $\mathrm{CD} 11 \mathrm{~b}^{+} \mathrm{F} 4 / 80^{+} \mathrm{CX} 3 \mathrm{CR} 1^{+}$resident renal macrophages exerted no obvious influence on UUOinduced renal fibrosis in either control or myeloid-specific $R B P-J$ knockout mice, suggesting that myeloid-specific Notch signaling might not regulate renal fibrosis through kidney resident macrophages. These results reminded us that Notch signaling might regulate monocyte-derived macrophages recruitment for renal fibrogenesis. Indeed, myeloid-specific $R B P-J$ knockout decreased while NIC over-expression increased the number of $\mathrm{CCR}^{+}$macrophages in fibrotic kidney, consistent with attenuated or aggravated renal fibrosis in these mice, respectively. This is also consistent with the finding by Lin et al., who showed that depletion of Ly6C $C^{\text {lo }}$ resident renal macrophages did not affect fibrosis whereas depletion of circulating monocytes and recruited Ly $6 \mathrm{C}^{\text {hi }}$ macrophages ameliorated renal fibrosis (Böttinger 2007; Lin et al., 2009). However, a recent study by Bettie et al has suggested that circulating monocytes-derived macrophages and liver resident macrophages share many common features and might have similar functions regardless of their origin (Beattie et al., 2016). Further investigations employing more precise lineage tracing and gene targeting mice, such as $C C R 2^{-1-}$ (Yona et al., 2013) and CX3CR $1^{\text {GFP }}$ transgenic (Seki et al., 2009) on the Notch deficient or activated background, are required to elucidate the cellular mechanism(s) for Notch signaling to regulate macrophages in renal fibrosis.

Inflammatory monocytes released from BM are recruited to inflammation sites and then differentiate into macrophages, followed by polarized activation upon local immunomicroenvironment (Yona et al., 2013). Notch signaling has been well demonstrated to participate in terminal differentiation, activation and polarization of macrophages in various disease models (Wang et al., 2010; Williams et al., 2010; Zhang et al., 2010; Xu et al., 2012; Franklin et al., 2014; Zhao et al., 2016). In the UUO-induced renal fibrosis, although it has been reported that different polarized macrophages, especially $\mathrm{M} 1$ and $\mathrm{M} 2$ macrophages, regulate the development of renal fibrosis by different mechanism (Vernon et al., 2010), in our study it appeared that both M1 and M2 types of macrophage activation were compromised by Notch blockade in macrophages upon UUO, suggesting that Notch signaling regulated macrophages activation, regardless of M1 or M2 macrophages during renal fibrogenesis. This phenomenon is consistent with our previous findings on Notch signaling regulation of macrophage activation in liver fibrosis (He et al., 2015). Recently, in murine liver fibrosis, Ranmachandran et al reports that one kind of new macrophage subsets are identified based on Ly6c expression, which are distinct from the M1/M2 paradigm, suggesting that more functional classification of macrophages subsets should be used to better represent their biology (Ramachandran et al., 2012). Indeed, Lin et al has found that Ly $6^{\text {hi }}$ and Ly6c ${ }^{\text {lo }}$ macrophages in kidney possess different function during renal fibrogenesis (Lin et al., 2009). Therefore, it might be possibility that Notch signaling regulates macrophage phenotype outside of M1/M2 classification in kidney and liver fibrosis. Functionally, RBP-J deficient macrophages exhibited reduced capacity of inducing EMT of tubular epithelial cells, due to less pro-fibrotic factor TGF- $\beta$ secretion in the fibrotic kidney and kidney macrophages. In addition to induce EMT, TGF- $\beta$ is reported to up-regulate the expression of CCL2 in macrophages and then promote monocyte recruitment and macrophage accumulation (Border and Noble, 1994; Qi et al., 2006). Therefore, it is reasonable to speculate that the less monocyte recruitment and macrophage infiltration in RBP-J cKO fibrotic kidney may caused by the reduced TGF- $\beta$ secretion through down-regulation of CCL2 expression in macrophages. Moreover, Franklin has reported that inflammatory monocytes are unable to differentiate into tumor associated macrophages in the absence of RBP-J by using $C D 11 c^{C r e} R B P-J / f$ PyMT mice (Franklin et al., 2014), this result may also partly explain our findings why less macrophages were accumulated in fibrotic kidney in $L y z^{\mathrm{Cre}} R B P-J^{f f f}$ mice.

In summary, our data have unveiled that Notch signaling regulates macrophage in renal fibrosis at two levels, namely the CCR2-mediated monocyte recruitment and the local macrophage activation (Fig. S8). These findings are of potential significance for establishing new therapeutic strategies for renal fibrosis in CKD. However, it should be cautious considering the spatial- and temporal-specific roles of Notch signaling in renal fibrosis. Notch signaling regulates EMT directly in several types of epithelial cells ( $\mathrm{Li}$ et al., 2013). Notch signaling is also a critical regulator of pericytes, which have been highlighted as an important source of myofibroblasts during renal fibrosis by recent studies (Duffield, 2014; Tattersall et al., 2016). Even in the macrophage compartment, sub-populations of macrophage with different origins and activation avenues likely exhibit different functions in renal fibrosis of different stages (Kitagawa et al., 2004; Nishida et al., 2005; Wang et al., 2007; Henderson et al., 2008). Specifically Notch-targeted therapies in macrophages might be a useful tool to overcome these obstacles for renal fibrosis treatment.

\section{MATERIALS AND METHODS}

Animals

Mice were maintained in the specific pathogen free (SPF) condition on the C57BL/6 background. Mice carrying Lyz2-Cre transgene (Clausen et al., 1999) (Stock \# 019096, The Jackson Laboratory) were crossed with RBP-J-floxed (RBP-Jf) (Han et al., 2002) mice or the ROSA-Stop-floxed-NIC (STOPf-NIC, a gift from $\mathrm{HL} \mathrm{Li)} \mathrm{to} \mathrm{obtain}$ $L y z 2-C r e / R B P-J^{+/ f}$ (Contrl) and Lyz2-Cre/RBP-J/f $(R B P-J$ cKO) mice (He et al., 2015), or Lyz-Cre (Contrl) and Lyz2-Cre/Stop ${ }^{f}-N I C\left(N I C^{C A}\right)$ mice (Zhao et al., 2016). Mice were genotyped by using PCR with the mouse tail DNA as a template. All primers were listed in Table S1. All animal experiments were approved by the Animal Experiment Administration Committee of the Fourth Military Medical University. All institutional and national guidelines for the care and use of laboratory animals were followed. 


\section{The mouse UUO model}

The mouse UUO model was established as described (Vielhauer et al., 2001; Chevalier et al., 2009). Briefly, mice were anesthetized with pentobarbital sodium (40 mg/kg) injected intraperitoneally (i.p.). A flank incision was made and the left ureter was ligated with 4-0 silk suture at two points and cut between the ligatures in order to prevent retrograde urinary tract infection. Both of the obstructed (Obstr) and contralateral (Contra) kidneys were harvested on day 7 or 14 after the ureteral ligation for further analyses. At least 6 pairs of mice were analyzed for each assay.

\section{Isolation of mouse kidney leukocytes and tubular epithelial cells}

Kidney cell suspensions were prepared as previously described (Kitamoto et al., 2009). Kidneys were dissected and dissociated in Hank's balanced salt solution (HBSS) containing $2.0 \mathrm{mg} / \mathrm{mL}$ collagenase IV (Sigma-Aldrich, St. Louis, MO) and $200 \mu \mathrm{g} / \mathrm{mL}$ DNase I (Sigma) for $30 \mathrm{~min}$ at $37{ }^{\circ} \mathrm{C}$ with intermittent agitation. Single cell suspensions were washed twice in HBSS. Following erythrocyte lysis, cells were washed twice again before further analyses.

For the isolation of kidney tubule epithelial cells, C57BL/6 mice (4-weeks old) were anesthetized and sacrificed. Kidneys were immediately removed and placed in ice-cold HBSS. The renal cortices were dissected visually and sliced into pieces of $1 \mathrm{~mm}$ in width, and transferred into $10 \mathrm{~mL}$ HBSS containing collagenase IV for each kidney. Tissues were incubated at $37^{\circ} \mathrm{C}$ with rotating at $70 \mathrm{rpm}$ for $30 \mathrm{~min}$. After that, Dulbeccoo's modified Eagle's medium (DMEM) containing $10 \%$ fetal bovine serum (FBS) was added to inactivate the enzymes. The tubule cell suspensions were then passed through a 200-mesh sieve to remove tissue debris, followed by centrifuge at 1,200 rpm for $5 \mathrm{~min}$, and resuspended in $10 \mathrm{~mL}$ DMEM/ $\mathrm{F}-12$ culture media containing $0.01 \mathrm{mg} / \mathrm{mL}$ recombinant human epidermal growth factor (rhEGF) and $10 \%$ FBS and antibiotics. Cells were cultured in 6 -well plates at $37{ }^{\circ} \mathrm{C}$ in $95 \%$ air- $5 \% \mathrm{CO}_{2}$.

\section{Flow cytometry}

Cells were re-suspended in FACS buffer (PBS containing 2\% FCS and $0.05 \% \mathrm{NaN}_{2}$ ) and pre-incubated with anti-rat $\mathrm{Fc}$ receptor (CD16/ 32) for $10 \mathrm{~min}$. And then cells were stained with Alexa Fluor 488 antimouse F4/80, APC anti-mouse CD11b, PE anti-mouse CCR2, APC anti-mouse CX3CR1, APC anti-mouse Ly6C, Biotin Ly6G and Avidin $\mathrm{PE}$. The detailed information for each antibody was listed in Table S2. FACS analysis was performed using a FACSCalibur ${ }^{\mathrm{TM}}$ flow cytometer (BD Immunocytometry Systems, Franklin Lakes, NJ). Data were analyzed with the Flowjo vX.06 software (Flowjo, LLC, Ashland, OR). Cell sorting was performed using a CytoFLEX flow cytometer (Beckman Coulter Life Sciences, IN). Dead cells were excluded by propidium iodide $(\mathrm{PI})$ staining.

Depletion of tissue resident macrophages with liposome-encapsulated clodronate

Clodronate (Sigma-Aldrich) was encapsulated in liposomes (CLs) as described (Van Rooijen and Sanders, 1994). Liposomes-encapsulated PBS (PLs) was used as a control. Mice were injected intravenously (i.v.) with $200 \mu \mathrm{L}$ of CLs or PLs on 2 consecutive days, followed by one more injection $48 \mathrm{~h}$ after the second one, and then subjected to UUO according to the protocol of macrophage depletion (Kitamoto et al., 2009).

\section{In vivo labeling with bromodeoxyuridine (BrdU)}

Mice were subjected to UUO, and injected i.p. with $\mathrm{BrdU}(1.2 \mathrm{mg} / 25 \mathrm{~g}$ of body weight) $2 \mathrm{~h}$ after the operation. The same BrdU injection was repeated every two days until the mice were sacrificed on day 7 . The kidney leukocytes were isolated and stained for BrdU incorporation in macrophages with APC anti-mouse BrdU (Biolegend) and Alexa Fluor 488 anti-mouse F4/80 for further FACS assay.

\section{Cell culture and transfection}

BM-derived macrophages (BMDMs) were isolated and cultured as previously described (Wang et al., 2010). In some case, kidney macrophages were sorted from the fibrotic kidney of RBP-J CKO and control mice by FACS, and then the sorted macrophages were counted and equal number of macrophages $\left(1 \times 10^{5}\right)$ from the RBP$J$ cKO and control mice were cultured in 48-well plate for $12 \mathrm{~h}$, followed by collection of supernatants as macrophage-derived conditional medium (CM) for further study.

The coding region of the murine CCL2 CDNA was amplified by PCR using primers (Forward: GCGAATTCAATGCAGGTCCCTGT CATGCTTCT, Reverse: GCGTCGACCTAGTTCACTGTCACACTG GTCA) with a mouse cDNA library as a template. The CCL2 gene was inserted into pCMV1-Flag to construct pCMV1-Flag-CCL2. HEK293 cells (ATCC) were cultured in DMEM (Invitrogen) supplemented with $10 \%$ FBS, $2 \mathrm{mmol} / \mathrm{L}$ L-glutamine, $100 \mathrm{U} / \mathrm{mL}$ penicillin and $100 \mu \mathrm{g} / \mathrm{mL}$ streptomycin. For transfection, cells were seeded in 24-well plates and transfected with pCMV1-Flag-CCL2 or pCMV1Flag using Lipofectamin $2000^{\mathrm{TM}}$ (Invitrogen, Carlsbad, CA) following the manufacturer's protocol. Cells were used for further experiments $24 \mathrm{~h}$ after the transfection.

\section{Cell migration assay}

BMDMs $\left(1.5 \times 10^{5}\right)$ were plated in the upper chamber of the transwell chambers with an $8 \mu \mathrm{m}$ polycarbonate filter (Millipore, Darmstadt, Germany) in DMEM with $10 \%$ FBS. HEK293 cells $\left(1.5 \times 10^{6}\right)$ transfected with $p C M V 1-$ Flag-CCL2 or pCMV1-Flag were plated in the lower chamber. Cell migration was allowed for $3.5 \mathrm{~h}$ by incubation at $37{ }^{\circ} \mathrm{C}$, and macrophages migrating to the lower chamber were counted under a microscope. Five randomly selected fields were counted as migrating cell number of each insert if not specified. Every experiment was repeated for at least three times with triplicates.

\section{Histology}

H\&E staining and Masson's trichrome and Sirius Red and $\alpha-S M A$ staining were performed following standard protocols ( $\mathrm{He}$ et al., 2015). The area of fibrosis after Masson's staining and Sirius Red staining, and $\alpha-\mathrm{SMA}^{+}$area were analyzed using the WinROOF image processing software (Olympus, Tokyo, Japan). At least 10 digitized images of the renal cortex were analyzed for each sample, and the percentage area of positive staining per field was evaluated. Images were taken under a microscope (BX51, Olympus) with a CCD camera (DP70, Olympus). 
For immunofluorescence, tissue sections (2-3 $\mu \mathrm{m}$ cryostat sections) were prepared according to standard procedures. The primary antibodies included anti-mouse F4/80. The secondary antibodies included biotinylated goat anti-rat $\lg G(\mathrm{H}+\mathrm{L})$ and Alexa 594 anti-rat IgG. DyLight 488 streptavidin was used for further staining with biotinylated antibodies. The detailed antibody information was listed in Table S2. Nuclei were counter-stained with Hoechst 33258 (Sigma). Images were taken under a fluorescence microscope (BX51, Olympus) or a laser scanning confocal microscope (FV1000, Olympus).

\section{RNA extraction and qRT-PCR}

Total RNA was prepared using the TriZol reagent (Invitrogen) according to the manufacturer's instructions. After reverse transcription, real-time PCR was performed using the SYBR Premix EX TaqTM II kit (Takara, Dalian, China) and the ABI PRISM 7500 realtime PCR system, with $\beta$-actin as an internal control. Primers for each gene were listed in Table S1.

\section{Western blotting analysis}

Western blotting was performed routinely, with primary antibodies against $\mathrm{E}$-cadherin, $\mathrm{N}$-cadherin, vimentin and $\beta$-actin. Horseradish peroxidase (HRP)-conjugated goat anti-rabbit lgG and goat antimouse IgG were used as the secondary antibodies. The detailed information for each antibody was listed in Table 2.

\section{Enzyme-linked immunosorbent assay (ELISA)}

The serum levels of TNF- $\alpha$ and TGF- $\beta$ were determined with commercial ELISA kits (eBioscience) following the recommended protocols.

\section{Chromatin immunoprecipiation (ChIP) assay}

The ChIP assay was performed using a kit (Merck Millipore, Billerica, MA) according to the manufacturer's instructions with monocytes from mouse BM. Freshly isolated cells were treated following the standard protocol, and fragmented chromatin preparations were immunoprecipitated with anti-RNA Pol II, anti-NIC or isotype control antibody. Genomic DNA was extracted from the collected immune complexes and analyzed using PCR with the primers targeting CCR2 promoter fragments (Table S1).

\section{Statistical analyses}

Images were processed using the Image Pro Plus 5.1 software (Media Cybernetics Inc, Bethesda, MA). Data were analyzed with Graph Pad Prism 5 software, version 5.0. Unpaired Student's $t$ test or paired $t$-test was used for the statistical analyses. The level of significance was set at $P<0.05$.

\section{ACKNOWLEDGEMENTS}

This work was supported by grants from the National Natural Science Foundation of China (Grant Nos. 81530018, 31371474, $81370811,31570878$ and 81300315$)$. This study was performed in the Graduates Innovation Center of Fourth Military Medical University.

\section{ABBREVIATIONS}

a-SMA, a-smooth muscle actin; BM, bone marrow; CKD, chronic kidney diseases; CLs, clodronate-liposomes; CM, conditional medium; CYLD, cylindromatosis; DAMPs, damage-associated molecular patterns; ECM, extracellular matrix; EMT, epithelial-tomesenchymal transition; MFI, mean fluorescence intensity; NIC, Notch intracellular domain; PTEpiC, proximal tubular epithelial cells; RBP-J, recombination signal binding protein-JK; SPF, specific pathogen free; UUO, unilateral ureteral obstruction.

\section{AUTHOR CONTRIBUTIONS}

Yali Jiang and Yuanyuan Wang conducted the main experiments and contributed equally to this work; Pengfei Ma and Dongjie An performed morphologic experiments; Junlong Zhao helped plasmids construction; Shiqian Liang maintained of mice and genotyping; Yuchen Ye did primary cell culture; Yingying Lu established UUO mouse mode and provided technical assistance for FACS; Peng Zhang performed H\&E staining; Xiaowei Liu, Hua Han and Hongyan Qin designed the experiments and wrote the paper.

\section{COMPLIANCE WITH ETHICAL GUIDELINES}

This article does not contain any studies with human subjects performed by any of the authors. All institutional and national guidelines for the care and use of laboratory animals were followed. Yali Jiang, Yuanyuan Wang, Pengfei Ma, Dongjie An, Junlong Zhao, Shiqian Liang, Yuchen Ye, Yingying Lu, Peng Zhang, Xiaowei Liu, Hua Han and Hongyan Qin declare that they have no conflicts of interest.

\section{OPEN ACCESS}

This article is distributed under the terms of the Creative Commons Attribution 4.0 International License (http://creativecommons.org/ licenses/by/4.0/), which permits unrestricted use, distribution, and reproduction in any medium, provided you give appropriate credit to the original author(s) and the source, provide a link to the Creative Commons license, and indicate if changes were made.

\section{REFERENCES}

Artavanis-Tsakonas S, Rand MD, Lake RJ (1999) Notch signaling: cell fate control and signal integration in development. Science 284:770-776

Beattie L, Sawtell A, Mann J, Frame TC, Teal B, de Labastida Rivera F, Brown N, Walwyn-Brown K, Moore JW, MacDonald S, Lim EK, Dalton JE, Engwerda CR, Macdonald KP, Kaye PM (2016) Bone marrow-derived and resident liver macrophages display unique transcriptomic signatures but similar biological functions. J Hepatol 65(64):758-768 
Border WA, Noble NA (1994) Transforming growth factor beta in tissue fibrosis. N Engl J Med 331:1286-1292

Böttinger EP (2007) TGF-beta in renal injury and disease. Semin Nephrol 27:309-320

Chen L, Al-Awqati Q (2005) Segmental expression of Notch and Hairy genes in nephrogenesis. Am J Physiol Renal Physiol 288: F939-F952

Chevalier RL, Forbes MS, Thornhill BA (2009) Ureteral obstruction as a model of renal interstitial fibrosis and obstructive nephropathy. Kidney Int 75:1145-1152

Clausen BE, Burkhardt C, Reith W, Renkawitz R, Förster I (1999) Conditional gene targeting in macrophages and granulocytes using LysMcre mice. Transgenic Res 8:265-277

Cochrane AL, Kett MM, Samuel CS, Campanale NV, Anderson WP, Hume DA, Little MH, Bertram JF, Ricardo SD (2005) Renal structural and functional repair in a mouse model of reversal of ureteral obstruction. J Am Soc Nephrol 16:3623-3630

Duffield JS (2014) Cellular and molecular mechanisms in kidney fibrosis. J Clin Invest 124:2299-2306

Falke LL, Gholizadeh S, Goldschmeding R, Kok RJ, Nguyen TQ (2015) Diverse origins of the myofibroblast-implications for kidney fibrosis. Nat Rev Nephrol 11:233-244

Franklin RA, Liao W, Sarkar A, Kim MV, Bivona MR, Liu K, Pamer EG, Li MO (2014) The cellular and molecular origin of tumorassociated macrophages. Science 344:921-925

Ginhoux F, Guilliams M (2016) Tissue-resident macrophage ontogeny and homeostasis. Immunity 44:439-449

Han H, Tanigaki K, Yamamoto N, Kuroda K, Yoshimoto M, Nakahata T, Ikuta K, Honjo T (2002) Inducible gene knockout of transcription factor recombination signal binding protein-J reveals its essential role in $T$ versus $B$ lineage decision. Int Immunol 14:637-645

Han Y, Masaki T, Hurst LA, Ikezumi Y, Trzaskos JM, Atkins RC, Nikolic-Paterson DJ (2008) Extracellular signal-regulated kinase dependent interstitial macrophage proliferation in the obstructed mouse kidney. Nephrology (Carlton) 13:411-418

Hashimoto D, Chow A, Noizat C, Teo P, Beasley MB, Leboeuf M, Becker CD, See P, Price J, Lucas D et al (2013) Tissue-resident macrophages self-maintain locally throughout adult life with minimal contribution from circulating monocytes. Immunity 38:792-804

He F, Guo FC, Li Z, Yu HC, Ma PF, Zhao JL, Feng L, Li WN, Liu XW, Qin HY et al (2015) Myeloid-specific disruption of recombination signal binding protein $\mathrm{J}$ k ameliorates hepatic fibrosis by attenuating inflammation through cylindromatosis in mice. Hepatology 61:303-314

Henderson NC, Mackinnon AC, Farnworth SL, Kipari T, Haslett C, Iredale JP, Liu FT, Hughes J, Sethi T (2008) Galectin-3 expression and secretion links macrophages to the promotion of renal fibrosis. Am J Pathol 172:288-298

$\mathrm{Hu}$ B, Phan SH (2016) Notch in fibrosis and as a target of anti-fibrotic therapy. Pharmacol Res 108:157-164

Hu M, Ou-Yang HF, Wu CG, Qu SY, Xu XT, Wang P (2014) Notch signaling regulates col1 $\alpha 1$ and col1 2 expression in airway fibroblasts. Exp Biol Med (Maywood). 239:1589-1596

Kalluri R, Weinberg RA (2009) The basics of epithelial-mesenchymal transition. J Clin Invest 119:1420-1428
Kitagawa K, Wada T, Furuichi K, Hashimoto H, Ishiwata Y, Asano M, Takeya M, Kuziel WA, Matsushima K, Mukaida N et al (2004) Blockade of CCR2 ameliorates progressive fibrosis in kidney. Am J Pathol 165:237-246

Kitamoto K, Machida Y, Uchida J, Izumi Y, Shiota M, Nakao T, Iwao H, Yukimura T, Nakatani T, Miura K (2009) Effects of liposome clodronate on renal leukocyte populations and renal fibrosis in murine obstructive nephropathy. J Pharmacol Sci 111:285-292

Kluth DC, Erwig LP, Rees AJ (2004) Multiple facets of macrophages in renal injury. Kidney Int 66:542-557

Le Meur Y, Tesch GH, Hill PA, Mu W, Foti R, Nikolic-Paterson DJ, Atkins RC (2002) Macrophage accumulation at a site of renal inflammation is dependent on the M-CSF/C-fms pathway. J Leukoc Biol 72:530-537

LeBleu VS, Taduri G, O'Connell J, Teng Y, Cooke VG, Woda C, Sugimoto H, Kalluri R (2013) Origin and function of myofibroblasts in kidney fibrosis. Nat Med 19:1047-1053

Li Y, Ma J, Qian X, Wu Q, Xia J, Miele L, Sarkar FH, Wang Z (2013) Regulation of EMT by Notch signaling pathway in tumor progression. Curr Cancer Drug Targets 13:957-962

Lin SL, Castano AP, Nowlin BT, Lupher ML, Lupher ML Jr, Duffield JS (2009) Bone marrow Ly6Chigh monocytes are selectively recruited to injured kidney and differentiate into functionally distinct populations. J Immunol 183:6733-6743

Ma FY, Sachchithananthan M, Flanc RS, Nikolic-Paterson DJ (2009) Mitogen activated protein kinases in renal fibrosis. Front Biosci (Schol Ed) 1:171-187

Meng XM, Wang S, Huang XR, Yang C, Xiao J, Zhang Y, To KF, Nikolic-Paterson DJ, Lan HY (2016) Inflammatory macrophages can transdifferentiate into myofibroblasts during renal fibrosis. Cell Death Dis 7:e2495

Miyajima A, Chen J, Lawrence C, Ledbetter S, Soslow RA, Stern J, Jha S, Pigato J, Lemer ML, Poppas DP et al (2000) Antibody to transforming growth factor-beta ameliorates tubular apoptosis in unilateral ureteral obstruction. Kidney Int 58(56):2301-2313

Monsalve E, Ruiz-García A, Baladrón V, Ruiz-Hidalgo MJ, SánchezSolana B, Rivero S, García-Ramírez JJ, Rubio A, Laborda J, Díaz-Guerra MJ (2009) Notch1 upregulates LPS-induced macrophage activation by increasing NF-kappaB activity. Eur $\mathrm{J}$ Immunol 39:2556-2570

Nikolic-Paterson DJ, Wang S, Lan HY (2014) Macrophages promote renal fibrosis through direct and indirect mechanisms. Kidney Int Suppl 4:34-38

Nishida M, Okumura Y, Fujimoto S, Shiraishi I, Itoi T, Hamaoka K (2005) Adoptive transfer of macrophages ameliorates renal fibrosis in mice. Biochem Biophys Res Commun 332:311-316

Qi W, Chen X, Polhill TS, Sumual S, Twigg S, Gilbert RE, Pollock CA (2006) TGF-beta1 induces IL-8 and MCP-1 through a connective tissue growth factor-independent pathway. Am J Physiol Renal Physiol 290:F703-F709

Ramachandran P, Pellicoro A, Vernon MA, Boulter L, Aucott RL, Ali A, Hartland SN, Snowdon VK, Cappon A, Gordon-Walker TT et al (2012) Differential Ly-6C expression identifies the recruited macrophage phenotype, which orchestrates the regression of murine liver fibrosis. Proc Natl Acad Sci USA 109:E3186-E3195 
Seki E, de Minicis S, Inokuchi S, Taura K, Miyai K, van Rooijen N, Schwabe RF, Brenner DA (2009) CCR2 promotes hepatic fibrosis in mice. Hepatology 50:185-197

Stamatiades EG, Tremblay ME, Bohm M, Crozet L, Bisht K, Kao D, Coelho C, Fan X, Yewdell WT, Davidson A et al (2016) Immune monitoring of trans-endothelial transport by kidney-resident macrophages. Cell 166:991-1003

Tang Y, Urs S, Liaw L (2008) Hairy-related transcription factors inhibit Notch-induced smooth muscle alpha-actin expression by interfering with Notch intracellular domain/CBF-1 complex interaction with the CBF-1-binding site. Circ Res 102:661-668

Tattersall IW, Du J, Cong Z, Cho BS, Klein AM, Dieck CL, Chaudhri RA, Cuervo H, Herts JH, Kitajewski J (2016) In vitro modeling of endothelial interaction with macrophages and pericytes demonstrates Notch signaling function in the vascular microenvironment. Angiogenesis 19:201-215

Van Rooijen N, Sanders A (1994) Liposome mediated depletion of macrophages: mechanism of action, preparation of liposomes and applications. J Immunol Methods 174:183-193

Vernon MA, Mylonas KJ, Hughes J (2010) Macrophages and renal fibrosis. Semin Nephrol 30:302-317

Vielhauer V, Anders HJ, Mack M, Cihak J, Strutz F, Stangassinger M, Luckow B, Gröne HJ, Schlöndorff D (2001) Obstructive nephropathy in the mouse: progressive fibrosis correlates with tubulointerstitial chemokine expression and accumulation of CC chemokine receptor 2- and 5-positive leukocytes. J Am Soc Nephrol 12:1173-1187

Wang YP, Harris DC (2011) Macrophages in kidney disease. J Am Soc Nephrol 22:21-27

Wang Y, Wang YP, Zheng G, Lee VW, Ouyang L, Chang DH, Mahajan D, Coombs J, Wang YM, Alexander SI et al (2007) Ex vivo programmed macrophages ameliorate experimental chronic inflammatory renal disease. Kidney Int 72:290-299

Wang YC, He F, Feng F, Liu XW, Dong GY, Qin HY, Hu XB, Zheng $\mathrm{MH}$, Liang $\mathrm{L}$, Feng $\mathrm{L}$ et al (2010) Notch signaling determines the
M1 versus M2 polarization of macrophages in antitumor immune responses. Cancer Res 70:4840-4849

Wang S, Meng XM, Ng YY, Ma FY, Zhou S, Zhang Y, Yang C, Huang $X R$, Xiao J, Wang $Y Y$ et al (2016) TGF- $/$ /Smad3 signalling regulates the transition of bone marrow-derived macrophages into myofibroblasts during tissue fibrosis. Oncotarget 7:8809-8822

Williams TM, Little MH, Ricardo SD (2010) Macrophages in renal development, injury, and repair. Semin Nephrol 30:255-267

Wynn TA, Vannella KM (2016) Macrophages in tissue repair, regeneration, and fibrosis. Immunity 44:450-462

Xu H, Zhu J, Smith S, Foldi J, Zhao B, Chung AY, Outtz H, Kitajewski J, Shi C, Weber S et al (2012) Notch-RBP-J signaling regulates the transcription factor IRF8 to promote inflammatory macrophage polarization. Nat Immunol 13:642-650

Yamasaki R, Lu H, Butovsky O (2014) Differential roles of microglia and monocytes in the inflamed central nervous system. J Exp Med 211:1533-1549

Yona S, Kim KW, Wolf Y, Mildner A, Varol D, Breker M, Strauss-Ayali D, Viukov S, Guilliams M, Misharin A et al (2013) Fate mapping reveals origins and dynamics of monocytes and tissue macrophages under homeostasis. Immunity 38:79-91

Zavadil J, Böttinger EP (2005) TGF-beta and epithelial-to-mesenchymal transitions. Oncogene 24:5764-5774

Zhang W, Xu W, Xiong S (2010) Blockade of Notch1 signaling alleviates murine lupus via blunting macrophage activation and M2b polarization. J Immunol 184:6465-6478

Zhao JL, Huang F, He F, Gao CC, Liang SQ, Ma PF, Dong GY, Han H, Qin HY (2016) Forced activation of Notch in macrophages represses tumor growth by upregulating miR-125a and disabling tumor-associated macrophages. Cancer Res 76:1403-1415

Zigmond E, Samia-Grinberg S, Pasmanik-Chor M, Brazowski E, Shibolet O, Halpern Z, Varol C (2014) Infiltrating monocytederived macrophages and resident kupffer cells display different ontogeny and functions in acute liver injury. J Immunol 193:344353 